\title{
EL IMPACTO TERRITORIAL DEL USO AGRÍCOLA Y TURÍSTICO DEL LITORAL: EVOLUCIÓN DE LOS CAMBIOS DE USO DEL SUELO EN LAS CUENCAS LITORALES DEL SUR DE LA REGIÓN DE MURCIA $(1956-2013)$
}

\author{
Daniel Ibarra Marinas \\ Francisco Belmonte Serrato \\ José Rubio Iborra \\ Departamento de Geografía. Universidad de Murcia \\ adaniel.ibarra@um.es, franbel@um.es
}

\section{RESUMEN}

Las áreas litorales mediterráneas han experimentado importantes transformaciones desde mediados del siglo pasado. Este trabajo analiza los cambios en los usos del suelo de las cuencas del litoral septentrional de la Región de Murcia, durante el periodo 1956-2013. Se ha elaborado una matriz de transición que contiene los cambios entre las coberturas. Los resultados muestran una evolución en la agricultura desde el secano tradicional al regadío con vocación de mercado. Los invernaderos, inéditos en 1956, pasan a ocupar una extensión significativa, a costa de los cultivos leñosos de secano tradicionales. Las áreas urbanas crecen debido al auge del turismo y a la necesidad de edificaciones para servicios agrícolas.

Palabras clave: Usos del suelo, litoral, SIG, agricultura intensiva.

\section{ABSTRACT}

The Mediterranean coastal areas have undergone major transformations since the middle of last century. This study analyzes changes in land use in the watersheds of the

Fecha de recepción: mayo 2015.

Fecha de aceptación: febrero 2016. 
southern coast of the Region of Murcia, between 1956 and 2013. We has developed a transition matrix containing the changes between the land covers. The results show an evolution in agriculture from traditional rainfed to irrigated market orientation. The greenhouses, unknow in 1956, come to occupy a significant extent, against the traditional upland woody. Urban areas are growing due to the growth of tourism and the need for buildings for agricultural services.

Keywords: Land uses changes, coastal areas, GIS, intensive agriculture.

\section{INTRODUCCIÓN}

Las actividades humanas han sido reconocidas como las principales fuerzas que transforman la biosfera, así como responsables de la mayor parte de los cambios contemporáneos en los paisajes actuales (Skole et al. 1994; Kummer y Turner 1995; Jansen y di Gregorio 2002). Estos cambios se encuentran en el centro de atención de la investigación desde finales de los noventa (Bocco et al. 2001), dado que este tipo de estudios proporciona la base para conocer las tendencias de procesos tales como la pérdida de la biodiversidad de una región determinada (Lambin et al. 2001), que en términos generales implican la pérdida de hábitat, diversidad biológica, servicios ambientales, la deforestación, la degradación y la erosión del suelo (Matson et al, 1997; Alonso Sarría et al, 2010) y en definitiva, la capacidad productiva de los ecosistemas (Dunjó et al. 2003). Las herramientas disponibles para el procesado de datos espaciales han facilitado y mejorado la obtención y tratamiento de los datos espaciales acompañando a otros medios como muestreos o encuestas. Los censos agrarios y los inventarios forestales (Peña et al., 2005) son otros de los instrumentos para evaluar los cambios en las coberturas. En este sentido, uno de los ámbitos más dinámicos en cuanto a los cambios experimentados recientemente son las áreas litorales, pese a que es reconocida su fragilidad a nivel universal, se trata de uno de los espacios que mayores transformaciones han sufrido en busca de un objetivo, su rendimiento económico, sin antes tener presente su condición de espacio dinámico, cambiante y vulnerable (Mir, 2009). Los usos del suelo, y su expresión física como cubiertas superficiales, constituyen el principal elemento de modificación del paisaje en amplias zonas de los sistemas mediterráneos (Pascual Aguilar, 2004) y, muy especialmente en las áreas litorales. En este sentido, el litoral de Región de Murcia ha experimentado una evolución que, contraria a la preservación de dicha fragilidad, ha estado condicionada por determinados sucesos que han tenido especial trascendencia en el devenir de estas áreas de especial interés ambiental, constituyendo un ejemplo paradigmático para analizar estos cambios.

Los cambios de uso del suelo en la Región de Murcia, así como en el resto de la cuenca mediterránea, están en gran parte vinculados a la dinámica socioeconómica (López-Bermúdez et al., 1995, Kosmas et al., 2002, Alonso et al., 2010), que aceleró la promoción residencial. El proceso de urbanización contó con el apoyo del gobierno regional, materializado en la política turística y, sobre todo, en el urbanismo y ordenación del territorio. La Región de Murcia se dotó de una normativa en materia de suelo que posibilitó y favoreció el crecimiento de este tipo de oferta. En concreto la Ley del Suelo (Ley 1/2001) abrió cla- 
ras oportunidades al fomento de los complejos residenciales (Vera, 2005). No obstante en la región de Murcia, esa dinámica reciente viene a incrementar los efectos de tres actividades que ya llevaban tiempo provocando cambios de usos del suelo en el litoral:

Por un lado la minería, que durante los últimos años del siglo XIX y primera década del siglo XX, alteró grandes sectores de las sierra litorales de la Unión, Cartagena y Mazarrón, aunque sin efectos apreciables en las costas; efectos que sí provocó la reactivación de esta actividad (esta vez a cielo abierto) en la sierra minera de Cartagena-La Unión en los años 80 del siglo XX, cambiando el paisaje de forma abrupta y provocando el aterramiento completo de la Bahía de Portmán; por otro lado, desde los años 60 se ha venido produciendo un proceso urbanizador en el área de la laguna litoral del Mar Menor, que ha llevado a la urbanización casi completa de la restinga de La Manga y del 80\% de la costa de la laguna y, por último, la llegada del agua procedente del río Tajo, a través del Trasvase Tajo-Segura en los primeros años de la década de los 80, que provocó un cambio radical en la agricultura de las zonas regables del Trasvase y que poco a poco se extendió a otras zonas, sobre todo del litoral sur, cuyos recursos hídricos procedían de otras fuentes, que llevaron a importantes cambios en los usos del suelo y medioambientales; hasta el punto de afectar incluso a los procesos de erosión costera.

Estos cambios se traducen principalmente en un incremento significativo de la agricultura intensiva, orientada al mercado, tanto de frutales de regadío como de hortalizas; la ocupación agrícola de los amplios cauces arenosos de las ramblas litorales; el aumento del sellado del suelo con superficies de invernadero dedicados a cultivos hidropónicos y el aumento de las superficies artificiales (urbano, industrial y pantanos de riego).

El objetivo de este trabajo es estudiar la evolución de los importantes cambios de usos del suelo acaecidos en el litoral sur de la Región de Murcia entre 1956 y 2013, en relación con el cambio de paradigma de la agricultura murciana y el desarrollo turístico.

\section{II. ÁREA DE ESTUDIO}

El área de estudio comprende las cuencas litorales del sur de la Región de Murcia (Figura 1), cuenta con una superficie de 709,62 Km² (70.962 ha), que afectan a los municipios de Cartagena, Mazarrón, Lorca y Águilas. Que se extienden entre Punta Parda (al suroeste, en el límite con la provincia de la Comunidad Autónoma de Andalucía), hasta la Isla de la Torrosa (próxima al puerto de Cartagena). Se trata de un territorio donde los relieves litorales de las estribaciones meridionales de la Cordillera Bética, pertenecientes al Bético Interno, se alzan con una disposición de arcos concéntricos, cóncavos hacia el mar, definiendo pequeñas cuencas neógenas en las que se mezclan depósitos marinos y continentales que contrastan con los relieves metamórficos.

El clima de esta zona es el más árido de la región de Murcia, con precipitaciones medias anuales que oscilan entre $282 \mathrm{~mm}$ en Mazarrón y $266 \mathrm{~mm}$ de Águilas, lo que la sitúa según el índice de aridez de Martonne, en el umbral entre los ambientes áridos y los semiáridos mediterráneos. Las temperaturas medias anuales oscilan entre los 18 y los $20^{\circ} \mathrm{C}$, con temperaturas medias mínimas entre 2 y $4^{\circ} \mathrm{C}$, con ausencia casi total de heladas, aunque las temperaturas medias máximas se suavizan mucho por la cercanía del mediterráneo, situándose entre los 35 y $37^{\circ} \mathrm{C}$. Estas condiciones, junto con las más de 3.000 horas de 
sol al año, explican el intenso aprovechamiento agrario y la expansión de los usos urbanos desde hace décadas. Se trata de un área colmada de recursos potenciales, que se han materializado cada vez que las sociedades que se han asentado allí, hacían uso de los mismos.

Figura 1

CUENCAS LITORALES DEL SUR DE LAREGIÓN DE MURCIA

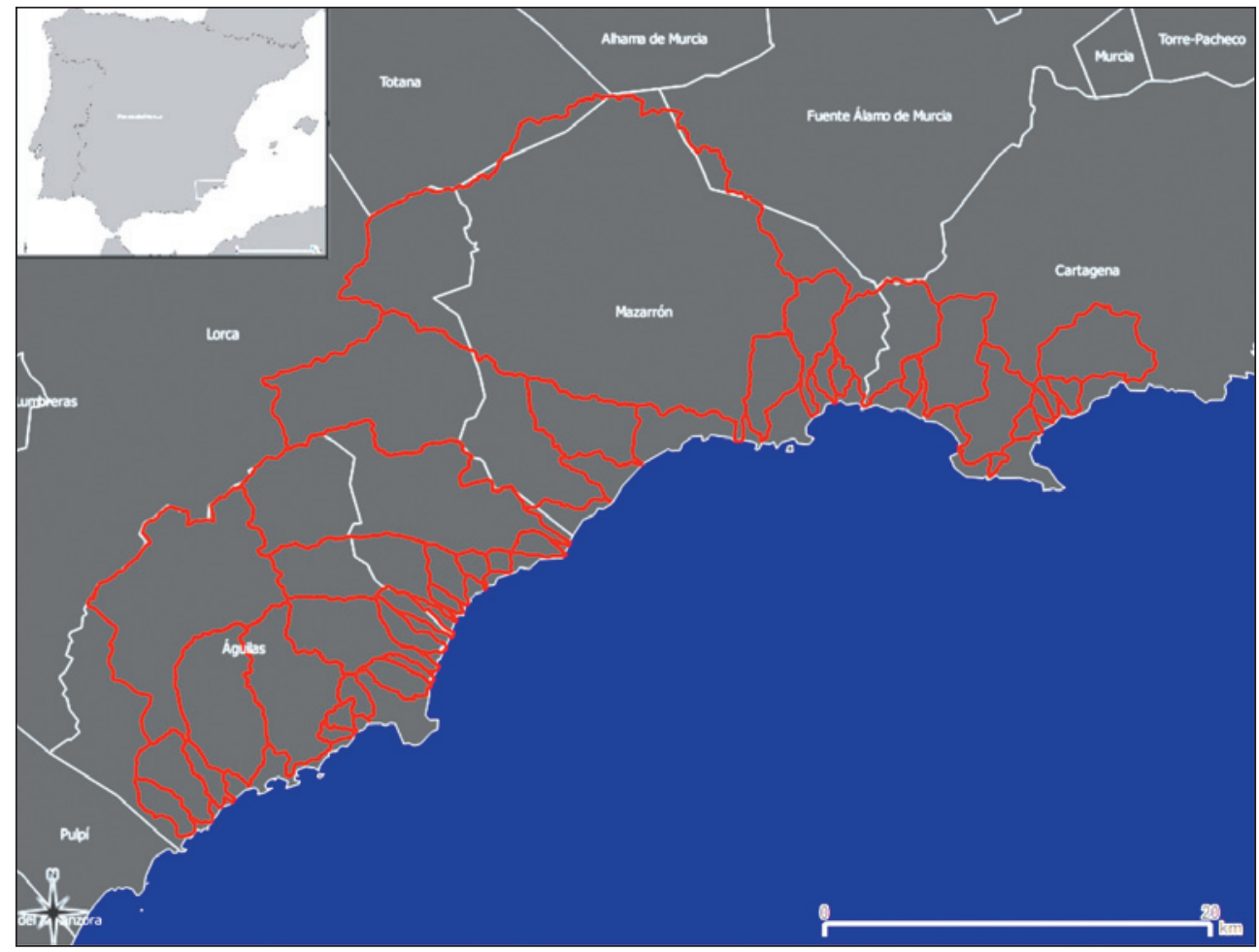

Fuente: elaboración propia.

\section{MATERIAL Y MÉTODOS}

\section{III.1. Material y herramientas}

Para la digitalización de los usos del suelo en 1956 se han utilizado las ortofotos USAF56, realizadas por el ejército de los Estados Unidos con una resolución geométrica de 1 metro. Los usos de 1981 se ha obtenido sobre la Ortofoto Regional de 1981. Los correspondientes a 2013 se han digitalizado sobre la fotografía aérea de máxima resolución del PNOA, que posee una resolución de 0,50 metros. Se ha dispuesto de las imágenes de 1956 y 1981 a través del servicio de WMS de la Infraestructura de Datos Espaciales de la Región de Murcia. La imagen correspondiente a 2013 se ha obtenido a través del centro de descargas del Instituto Geográfico Nacional. 
Los mapas a escala 1:25.000, paginas 976-III y 975-IV del Mapa Topográfico Nacional en formato papel han sido utilizados para apoyar la fotointerpretación. Los SIG tienen la capacidad de homogeneizar información procedente de diferentes fuentes y permiten el posterior tratamiento en un solo formato, por lo que se convierten en herramientas muy útiles para el estudio de las variaciones espaciales de los procesos geográficos (Pascual, 2004) Para la visualización, digitalización y tratamiento de datos se ha utilizado el software libre QGIS de Código Abierto bajo licencia GNU.

\section{III.2. Metodología}

El método que se ha utilizado para el análisis de los usos del suelo, siguiendo el ejemplo de otros trabajos, se ha basado en la identificación de los cambios en las componentes espacial y temática (Rosete Vergés et al., 2009) y en la representación de los procesos espaciotemporales con el fin de expresar las diferencias entre distintos momentos temporales para las distintas unidades (Gutiérrez y Gould, 2000).

Se han identificado las coberturas que definen los diferentes usos del suelo para cada año de estudio. Las características de la fotointerpretación han sido las siguientes:

- La unidad de trabajo es el polígono, que es la unidad espacial del terreno que presenta una cobertura homogénea.

- El modelo de datos para las coberturas solo reconoce el polígono como entidad con geometría propia. En el caso del viario se han utilizado polilíneas.

- Las coberturas se clasifican en:

o Tierras arables (básicamente cultivos hortícolas de regadío)

o Frutales de secano (árboles con un marco de plantación entre 8 y 10 m, generalmente a tresbolillo)

o Frutales de regadío (árboles con un marco de plantación entre 5 y 7 m, casi siempre en hileras)

o Invernaderos

o Láminas de agua (balsas de riego y otras superficies de agua)

o Zonas urbanas

o Zonas forestales

o Reforestaciones

o Otros usos (monte bajo, matorral, o erial a pastos)

- Se han obtenido dos niveles de viarios: autovías y carreteras.

La digitalización se ha llevado a cabo en una escala no superior a 1:5.000, mediante la que se han delimitado las distintas coberturas y usos del suelo correspondientes a los tres periodos (1956, 1981 y 2013) (Figura 2).

La digitalización no permite precisar entre tierras arables de regadío y de secano, si bien, sabemos que las condiciones de extrema aridez del área, no permiten el cultivo hortícola ni cerealista en secano, y, aunque este último se da, su presencia es mínima a causa de los bajísimos rendimientos que produce: por ello asumimos que el uso de tierra arable se corresponde, casi en su totalidad, con cultivos hortícolas de regadío (básicamente riego localizado). 
Figura 2

ÁREA PRÓXIMA A MAZARRÓN EN 1956, 1981 Y 2013
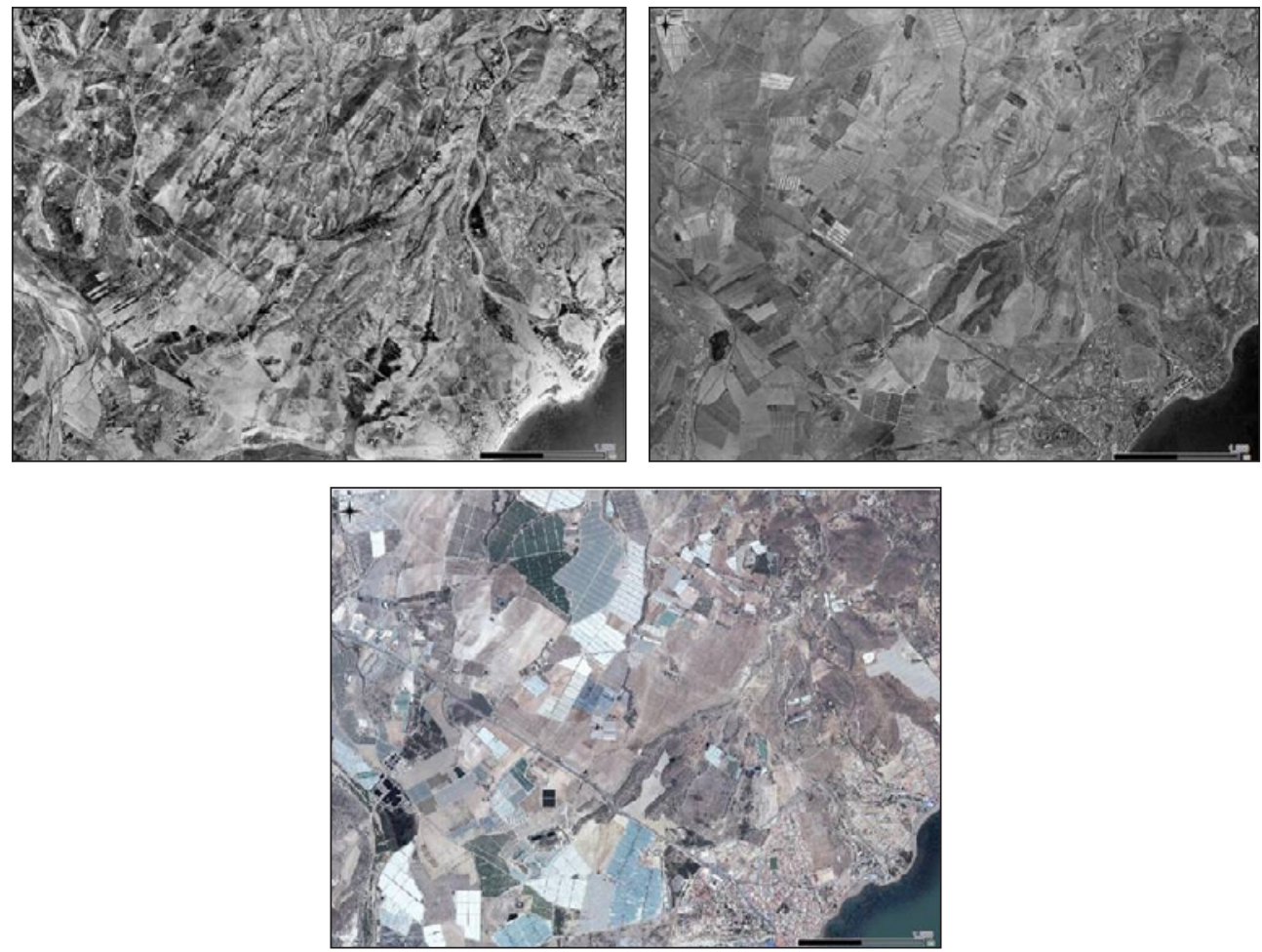

Fuente: elaboración propia.

Por su parte, la diferenciación entre los cultivos leñosos de regadío y secano, se puede realizar mediante la clasificación de estos por el marco de plantación del cultivo (distancia entre árboles) y su disposición. En secano, sobre todo en secanos con escasas precipitaciones como los que nos competen, los marcos de plantación son amplios, entre 8 y 10 metros (entre 100 y 180 árboles por hectárea) y generalmente plantados a tresbolillo, que permite un mejor aprovechamiento de la poca humedad del suelo. En regadío, al no existir competencia por el agua, se puede aumentar mucho la densidad de plantación, que dependiendo de los frutales y la variedad se planta con marcos rectangulares de 7X7 o 6X6 (de 200 a 240 árboles por hectárea), o en líneas de 7X6 o 6X5 (de 277 a 333 árboles por hectárea). Los invernaderos son fácilmente identificables. La clasificación "otros usos" recoge principalmente monte bajo y matorral, además de viarios principales y caminos de huerta.

Después de definir y obtener los usos, existen varios modelos de tasa de cambio para calcular la evolución de la cobertura, en este trabajo se ha utilizado la tasa de cambio de la FAO (1996) para cada año.

$$
\delta n=\left(\frac{S_{2}}{S_{1}}\right)^{1 / n}-1
$$


Donde $\mathrm{C}=$ Tasa de cambio, $\mathrm{S} 1=$ Superficie en la fecha $1, \mathrm{~S} 2=$ Superficie en la fecha 2 y $n=$ Número de años entre las diferentes fechas. El valor absoluto máximo de las tasas es 1.

\section{RESULTADOS}

\section{IV.1. Etapas de cambios}

Primera etapa (1956-1981)

En la primera etapa, las tendencias de cambio (Figuras 3 y 4) muestran un gran descenso de "otros usos", es decir, monte bajo, matorral, o erial a pastos, en favor del resto de usos; salvo el uso de frutales de secano que también descienden. Sin duda, su escasa rentabilidad, frente a una agricultura de regadío orientada al mercado es el factor clave de este descenso. De hecho, esta circunstancia se aprecia en que la Tierra arable aumenta entre 1956 y 1981, al mismo tiempo que desciende los frutales de secano. El aumento, alcanza las 5.282 ha durante el periodo 1956 y 1981, alcanzando este último año 11.327,52 ha, lo que indica que las grandes expectativas que se abren en la agricultura murciana con la puesta en funcionamiento del Trasvase Tajo-Segura, está convirtiendo terrenos nunca cultivados en tierra arable, es decir, en tierra preparada para la agricultura, muy probablemente de regadío en la medida en que haya disponibilidad de agua; pero también, se está produciendo un cambio de uso desde frutales de secano a tierra arable y a otros usos agrícolas de regadío que empiezan ya tímidamente en 1981 a tomar forma, como los invernaderos y los frutales de regadío.

Los primeros invernaderos en la Península Ibérica se instalaron a mediados de la década de los 60 (Tolón y Lastra , 2010), por lo que este uso es inexistente en 1956. Pero en 1981 en las cuencas litorales estudiadas se alcanzan ya las 519,23 ha.

En esta primera etapa, el área urbana aumenta muy poco. Se está apenas en los inicios del cambio de una agricultura tradicional a la agricultura intensiva orientada al mercado, que predominará en la etapa siguiente, de modo que todavía no se ha producido una atracción de población necesaria para las nuevas tareas agrícolas, ni tampoco se ha desarrollado una industria auxiliar (manipulación, embasado y transporte) que incrementará la necesidad de mano de obra y ocupara suelo agrícola. De modo que el aumento se debe exclusivamente al inicio de la actividad turística, que hace que las 94,86 ha de 1956 asciendan a 300 ha en 1981, en gran parte ligado al boom inmobiliario del periodo 1970-1974 (Rodríguez López, 2006).

Se detecta un importante aumento del uso forestal, el cual, en principio parece debido tanto a una "reforestación", como a recolonización espontánea de la vegetación arbórea sobre terrenos, o bien agrícolas abandonados o sobre monte bajo y matorral. En este periodo, la superficie forestal se multiplica por 5 pasando de 159 ha forestales de 1956 a 836 ha en 1981 .

Por su parte las balsas de riego, contabilizadas aquí como "láminas de agua" pasan de ocupar 34,39 ha en 1956 a 103,63 en 1981. Se trata, todavía en este periodo de construcciones de hormigón o de mampostería enlucida, muy distintas en tamaño y en tipo constructivo de las que se construirán en la etapa siguiente.

Por último la red de transporte no experimentó grandes cambios en esta etapa, más allá del asfaltado y mejora de algunos caminos y carreteras, pasando la superficie asfaltada de 352 Km en el año 1956, a sólo 400 Km en 1981. 
Figura 3

SUPERFICIE DE LOS USOS EN LOS AÑOS 1956, 1981 Y 2013

$\mathrm{TA}=$ tierra arable; $\mathrm{FS}=$ frutal secano; $\mathrm{FR}=$ frutal regadío; $\mathrm{IV}=$ invernadero; $\mathrm{ZU}=$ zona urbana;

$\mathrm{AG}=$ láminas de agua; $\mathrm{FO}=$ forestal; $\mathrm{REF}=$ reforestaciones; otros usos

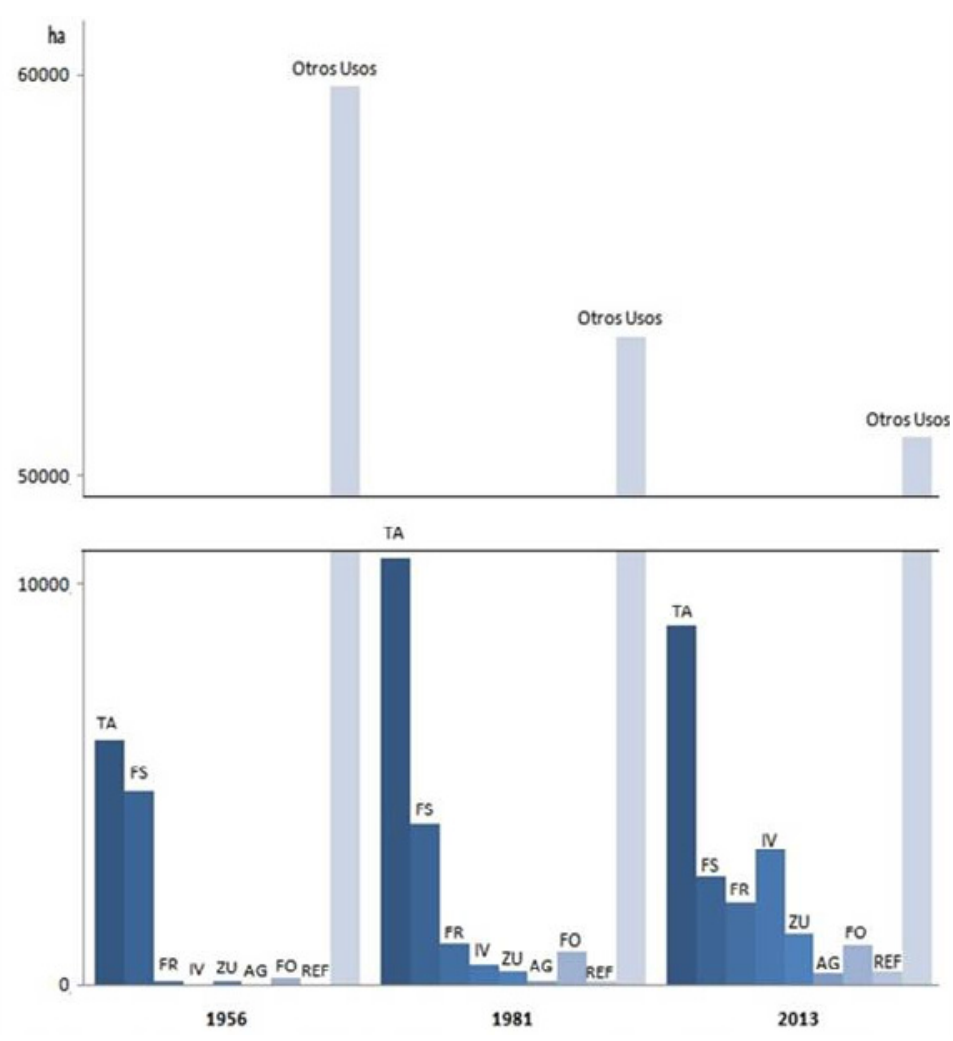

Fuente: elaboración propia.

\section{Segunda etapa (1981-2013)}

En la segunda etapa (Figuras 3 y 4), se mantiene el descenso, aunque algo menor que en la etapa anterior, de las tierras ocupadas por "otros usos"; los frutales de secano mantienen también su descenso, y, al contrario de la etapa anterior, descienden también las tierras arables en 2.261 ha, perdiendo el $43 \%$ del aumento experimentado en la etapa anterior. Todos estos descensos se ven compensados por el aumento de los invernaderos, que multiplican por 6.6 su superficie, pasando de 519 ha en 1981 a 3.409 ha en 2013, los frutales de regadío que multiplican por 8 su superficie (de 75,55 ha en 1956 a 6.137,46 ha en 2013). Los usos de frutal de regadío e invernadero han invadido espacios improductivos como los piedemontes (Belmonte Serrato et al., 2011; Romero Díaz et al., 2012), así como una gran superficie de secano tradicional (ver tabla 1 ). 
Además, en esta segunda etapa, también aumentan muy ligeramente la superficie forestal, seguramente porque continúa su recolonización en los terrenos menos propicios para la moderna agricultura, y también aparece un pequeño porcentaje de tierras reforestadas, seguramente en tierras de cultivo abandonadas acogidas a algunas ayudas a la forestación de tierras agrícolas. De las 836 ha en 1981, se pasa a 1001,41 ha de 2013, (165 ha más). La mayor parte de los cambios de usos de suelos forestales se distribuyen en las zonas más alejadas de la costa (Figura 6)

Se produce además un importante incremento de las áreas urbanas, uno de los cambios más fácilmente identificables en el litoral. Existen dos booms inmobiliarios posteriores a 1981, el de 1986-1991 y sobe todo, el del periodo 1997-2006, excepcional por su intensidad y su duración (Burriel, 2008) y acelerado por la mejora de la red de transportes que permiten que la superficie edificada pase de 300 ha en 1981 a $1.268,48$ ha en 2013 . Un total de 229,99 ha de tierra arable sufren esa transformación. El monte bajo y el matorral, recogidos en la clasificación "otros usos" es aprovechado para la expansión de 874,02 ha de edificaciones. Las áreas próximas a la costa son las que han experimentado un mayor crecimiento.

La distribución del incremento de las zonas urbanas posee dos características espaciales, la primera es su cercanía a la línea de costa, especialmente en las localidades de Águilas y Mazarrón. La segunda está ligada a la protección de la Marina de Cope. Esta área de gran importancia medioambiental fue declarada Parque Natural por la legislación de la Comunidad Autónoma de Murcia en la década de los noventa, por medio de la Ley de Ordenación y Protección del Territorio de la Región de Murcia de 1992. Mediante la Ley del Suelo de abril de 2001 de la Comunidad de Murcia, se permite su recalificación. Finalmente, en 2005 el Tribunal Constitucional declaró inconstitucional dicha ley tras un recurso. En la actualidad se encuentra incluido dentro de la ZEPA Sierra de la Almenara, Moreras y Cabo Cope (Belmonte Serrato et al., 2013) y está declarado también como LIC (Lugar de Importancia Comunitaria): LIC Calnegre y LIC-Cabo Cope.

En esta segunda etapa, la red de transporte mejora considerablemente, primero con la puesta en servicio de las autovías de Lorca-Águilas y Totana-Mazarrón, que unen los dos núcleos más importantes del litoral sur con la autovía del Mediterráneo (A7), y a partir del año 2007con la puesta en servicio de la autopista de peaje Cartagena-Vera, de recorrido esteoeste y la autovía entre Alhama de Murcia y Mazarrón que aumenta los accesos por autovía a la A7. De este modo los $400 \mathrm{Km}$ de carreteras asfaltadas en 1981 pasan $581 \mathrm{Km}$ en 2013, de los cuales 100 pertenecen a las autovías y autopista señaladas.

Las superficies de balsas de riego (lámina de agua) aumentaron de 103 ha en 1956 a 1.268,48 ha de 2013, multiplicando por 12 su superficie. La proliferación de esta cobertura, ligada a la expansión del regadío, dada la necesidad de optimizar las escasas disponibilidades hídricas para el desarrollo de una agricultura de alto rendimiento, almacenando los escasos recursos hídricos disponibles. El agua del trasvase no llegó al área litoral de la Región de Murcia de forma directa lo que aumentó el crecimiento de este tipo de embalses, a pesar de los problemas derivados de la pérdida de agua por evaporación. En las cuencas de las ramblas de Pastrana, Villalba y Ramonete, $\left(174 \mathrm{Km}^{2}\right)$ en sólo 50 ha de balsas, las pérdidas por evaporación se han estimado en $1 \mathrm{Hm}^{3}$ anual (Ibarra Marinas et al., 2014). Esta situación puede requerir en el futuro, la adopción de medidas para paliar esas pérdidas o la adopción de sistemas de riego orientados a la sostenibilidad de recursos hídricos (Martínez-Fernández y Esteve, 2010). 


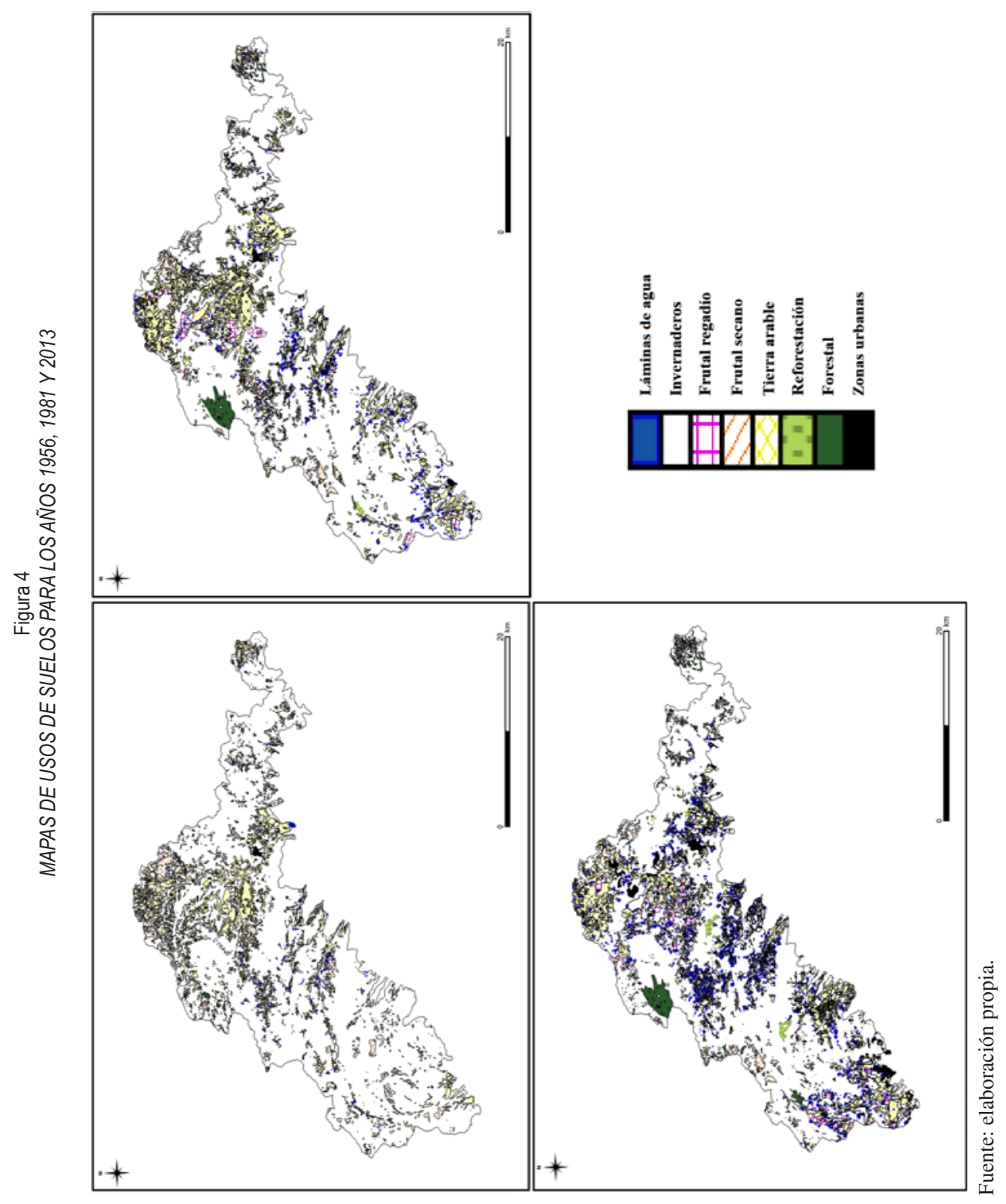




\section{IV.2. Cambios entre usos}

Se ha realizado una matriz de cambios (Tabla 1) para comparar entre los usos de suelo de 1956 y 2013. El uso de una matriz de cambios es de gran utilidad a la hora de contrastar los cambios en los diferentes usos (López et al. 2001, Aguayo et al. 2009). En este caso, contiene las variaciones entre tierra arable (TA), frutal regadío (FR), frutal secano (FS), invernadero (IV), láminas de agua (AG), zona urbana (ZU), forestal (FO), reforestaciones (REF) y otros usos.

El cruce de datos representa las transformaciones de las coberturas a lo largo del periodo de estudio. La suma de las filas y columnas representa las hectáreas totales de cada cobertura en el año 1956 y 2013, respectivamente. La lectura de los valores en el sentido de las columnas indica el cambio de cobertura de suelo ocurrido entre 1956 y 2013. Las celdas situadas en la diagonal de la matriz contienen la superficie de aquellas áreas que no experimentaron cambios durante el período.

La matriz de transición muestra la trasferencia de cambios entre usos en hectáreas, pero para clarificar mejor la situación se ha elaborado un diagrama de transiciones (Figura 5) que muestra el porcentaje de superficie perdida o ganada de cada uso con el resto de usos y consigo mismo (Aguayo et al., 2009). Para su elaboración se han agrupado los usos reforestación y forestal en un solo uso (forestales), así como los usos invernaderos y frutal de regadío, contenidos en el uso regadíos, Sólo se incluyen los porcentajes mayores a $1 \%$.

Tabla 1

MATRIZ DE CAMBIOS DE COBERTURAS EN HAS

\begin{tabular}{|lr|r|r|r|r|r|r|r|r|r|}
\cline { 2 - 13 } \multicolumn{1}{|c|}{} & \multicolumn{10}{|c|}{$\mathbf{2 0 1 3}$} \\
\hline Uso 1956 & \multicolumn{1}{|c|}{ TA } & \multicolumn{1}{c|}{ FR } & \multicolumn{1}{c|}{ FS } & IV & AWR & URB & FO & REF & Otros usos & Total 2013 \\
\hline TA & $\mathbf{2 . 0 3 6 , 1 6}$ & 11,88 & 353,25 & 0,00 & 0,83 & 1,51 & 0,00 & 0,00 & $6.540,67$ & $8.944,30$ \\
\hline FR & 509,68 & $\mathbf{7 , 1 5}$ & 247,92 & 0,00 & 0,08 & 0,53 & 0,00 & 0,00 & $1.258,59$ & $2.023,95$ \\
\hline FS & 0,33 & 2,12 & $\mathbf{9 1 2 , 8 4}$ & 0,00 & 0,08 & 0,37 & 0,02 & 0,00 & $1.812,17$ & $2.727,93$ \\
\hline IV & 645,11 & 3,97 & 353,24 & $\mathbf{0 , 0 0}$ & 0,11 & 0,44 & 0,00 & 0,00 & $2.389,02$ & $3.391,89$ \\
\hline AG & 25,63 & 0,00 & 15,50 & 0,00 & $\mathbf{0 , 3 4}$ & 0,08 & 0,00 & 0,00 & 259,11 & 300,66 \\
\hline URB & 229,96 & 1,95 & 89,78 & 0,00 & 1,59 & $\mathbf{7 9 , 0 0}$ & 0,00 & 0,00 & 942,92 & $1.266,24$ \\
\hline FO & 24,65 & 0,00 & 10,75 & 0,00 & 0,02 & 0,00 & $\mathbf{1 1 0 , 3 7}$ & 0,00 & 845,21 & 990,99 \\
\hline REF & 0,00 & 0,00 & 1,53 & 0,00 & 0,00 & 0,00 & 0,00 & $\mathbf{0 , 0 0}$ & 311,93 & 313,47 \\
\hline Otros usos & $2.623,13$ & 48,48 & $2.851,38$ & 0,00 & 14,08 & 7,84 & 48,53 & 0,00 & $\mathbf{4 5 . 3 3 0 , 6 6}$ & $51.003,06$ \\
\hline Total 1956 & $6.094,65$ & 75,54 & $4.836,20$ & 0,00 & 17,13 & 89,77 & 158,92 & 0,00 & $59.690,28$ & $\mathbf{7 0 . 9 6 2 , 4 9}$ \\
\hline
\end{tabular}

Fuente: elaboración propia.

En el periodo 1956-2013, 2.036 ha de tierra arable no experimentan cambios, lo que supone el 33,4 \% de la superficie en 1956, sin embargo, el $18 \%$ de este uso evoluciona hacia regadíos en el periodo 1956-2013, de esta manera, 509,68 ha de tierra arable se transforman en frutales de regadío y 645,11 ha se corresponden con invernaderos, debido principalmente al proceso de modernización de la agricultura. En cambio un $43 \%$ de este uso pasa a la cobertura "otros usos", lo que explica un proceso de abandono de tierras. 
El uso de frutales de regadío que, como se ha comentado experimenta un crecimiento muy importante en el periodo de estudio, gana terreno, sobre todo, al monte bajo y matorral (otros usos) recibiendo de ellos, 1.258,59 ha. La evolución de la agricultura tradicional a la de mercado se ve representada en las 247,92 ha de frutal de regadío y las 353,24 ha de invernadero procedentes de frutales de secano. Parte de este uso tradicional $(18,9 \%)$ permanece en 2013.

El 69,5\% de la superficie forestal de 1956, 110,37 ha, permanece como tal en 2013. El incremento de este uso procede principalmente del monte bajo y el matorral, que aporta 845,21 a bosque mediterráneo y 311,93 ha a nuevas reforestaciones.

En su evolución 1956-2013 las nuevas áreas urbanas proceden de todos los usos, salvo los invernaderos, y usos forestales. Las zonas urbanas de 1956 son las que menos transferencias hacen hacia otros usos, el $88 \%$ de la superficie construida permanece en 2013 . No obstante, hay una parte que se pierde. El $11 \%$ pasa a la cobertura "otros usos", esta transferencia ésta motivada por la construcción de autovías y por el abandono y posterior ruina de edificaciones en las zonas más alejadas de la costa.

Por último, el incremento de las balsas no se desarrollan donde se situaban las antiguas superficies de agua. Las balsas de 2013 requieren mayor espacio y esto hace que se abandonen el $82,2 \%$ de la superficie de sus antiguas ubicaciones. La cobertura "otros usos" transfiere 259,11 ha a este uso.

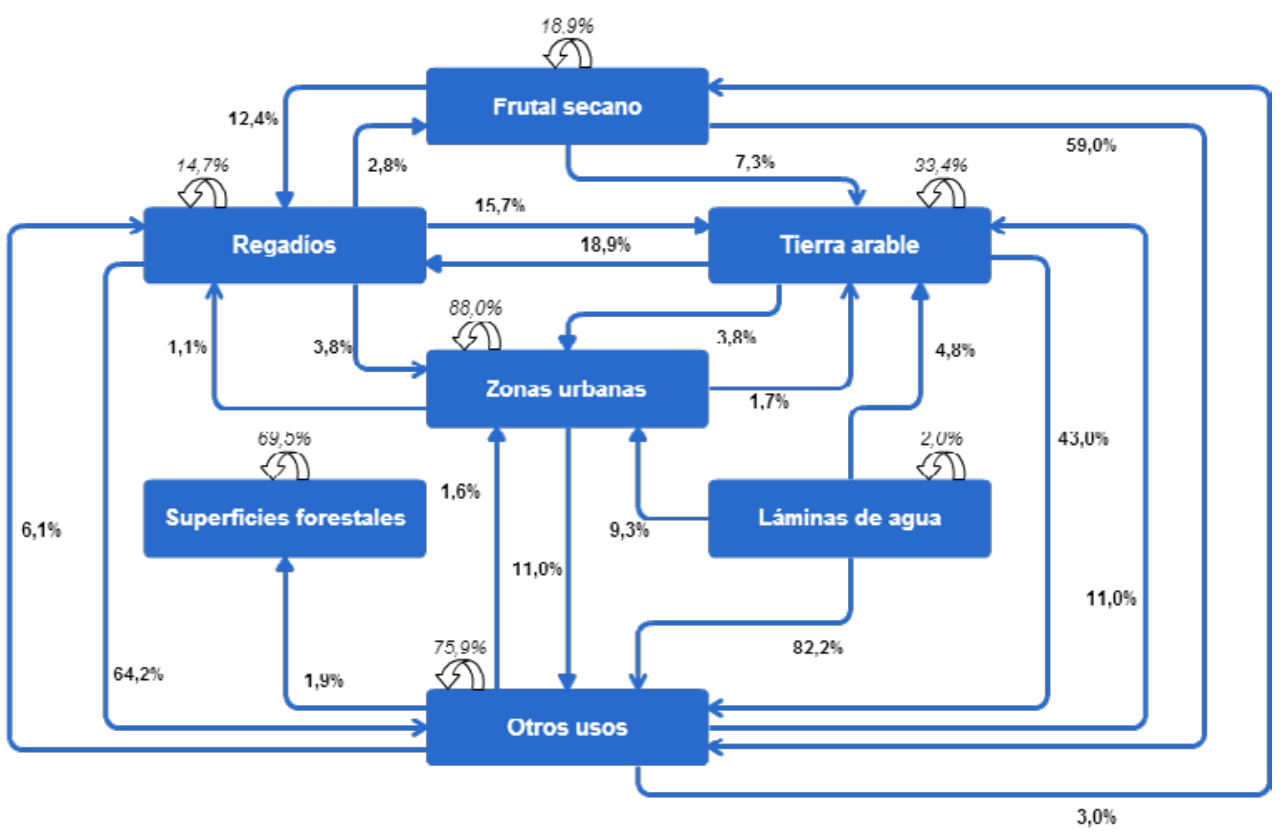

Fuente: elaboración propia. 
El mapa de detección de cambios de usos del suelo (Figura 6) es la representación cartográfica de la matriz de cambios. La mayor parte de los cambios se experimentan en las áreas situadas en las cuencas de las ramblas y parte de las áreas litorales, mientras las áreas correspondientes a las sierras y las áreas urbanas de 1956 conservan sus usos a lo largo del periodo de estudio. En conjunto, como puede apreciarse en la figura 6, los cambios de uso han afectado a 48.477 ha, el $68,3 \%$ de la superficie total.

Figura 6

MAPA DE DETECCIÓN DE CAMBIOS DE USOS DEL SUELO 1956-2013

(EN COLOR LA SUPERFICIE QUE HA CAMBIADO DE USO ENTRE 1956 Y 2013)

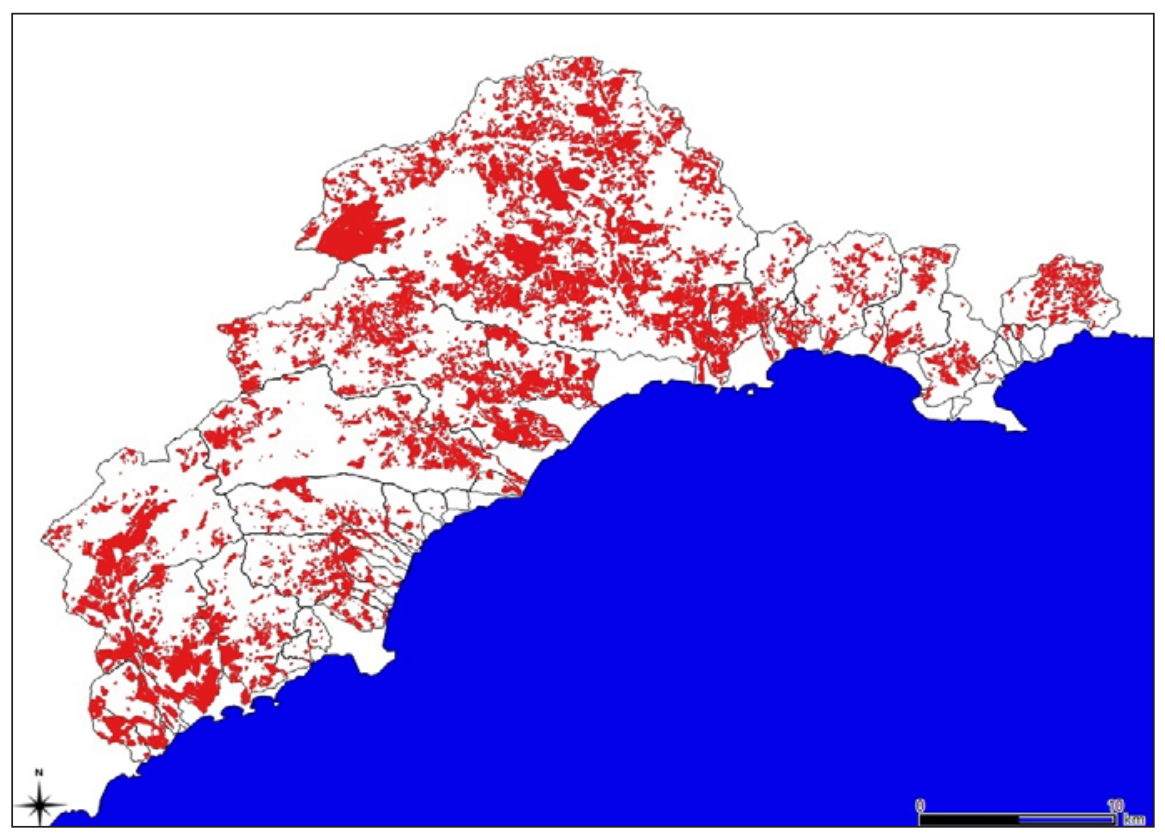

Fuente: elaboración propia.

\section{DISCUSIÓN Y CONCLUSIONES}

Muchos de los estudios de cambios de uso del suelo en el ámbito Mediterráneo ofrecen resultados similares. De esta manera, en el sur de la provincia de Alicante, las 26.000 ha de regadío de principios de los años setenta pasan a 59.940 ha en 2006 (Hernández Hernández y Morales Gil, 2008). En el Valle del Guadalhorce (Málaga) los cultivos de regadío ocupan ahora más de un 40\% del territorio (Rodrigo et al., 2014), la extensión porcentual de este uso es considerablemente mayor que en las cuencas del litoral de Murcia. Las tendencias de los cambios experimentados en las coberturas son similares, en el periodo de estudio 1991-2007, se observan procesos de urbanización y el retroceso de los cultivos de secano. La mayor parte de la superficie de este uso, está relacionada con el cultivo de almendros, no sólo en el área de estudio, también a nivel regional. En 2004 el almendro representaba el 3,6 \% de la 
superficie de cultivo en España (Calatrava Leyva, 2006), mientras en la Región de Murcia era del $12 \%$, a pesar del retroceso sufrido a partir de los años 80 . Pese a que el crecimiento del cultivo de almendro en la región se ha visto favorecido por las ayudas de la Política Agraria Comunitaria, en el área de estudio no han conseguido frenar su sustitución por el regadío, más rentable. El 12,5 \% del frutal de secano se convirtió en regadío en el periodo de estudio, mientras el $43 \%$ se transformó en otros usos (principalmente monte bajo) lo que sugiere un abandono generalizado de la actividad.

El Decreto de 25 da abril de 1953, se dispuso para reglamentar una mejor ordenación de los caudales que circulan por la cuenca del Río Segura para llevar su aprovechamiento al máximo, respetar los derechos adquiridos y utilizar los caudales regulados por los pantanos en construcción y construidos por el Estado, con el fin de conseguir una ampliación a nuevos regadíos de marcado interés nacional (Canales Martínez y Juárez Sanchez-Rubio, 1994). El establecimiento de los pantanos del Cenajo y Camarilla, construidos en 1957 y 1960 respectivamente, permitió que los volúmenes regulados en la Cuenca del Segura aumentaran en 233 Hm3/año. Así, el incremento del regadío se hizo posible gracias a una mayor disponibilidad de agua embalsada, que fue viable según determina el Decreto Orden Ministerial de 1953. El agua embalsada en la cuenca del segura y el abastecimiento del trasvase Tajo-Segura no afectaron directamente al área litoral de la región, pero sí produjo un efecto llamada apoyado por los factores edáficos y por las favorables condiciones climáticas del litoral, en especial la ausencia de heladas (Morales Gil, 2003). De esta manera, se comenzó a sentar una base para los futuros cambios de uso de suelo agrícola, orientando los cultivos tradicionales de secano a una agricultura intensiva de regadío.

En las últimas décadas, debido al auge del turismo, la superficie edificada ha crecido de forma significativa por el litoral mediterráneo. En la Comunidad Valenciana las zonas urbanas han experimentado un crecimiento de 16.557,24 ha en el periodo 1987-2000 (Gaja i Díaz, 2008) que supone un incremento de 30,3\%, para el periodo 1981-2013 en las cuencas del área de estudio el aumento ha sido del 42\%. En el Área Metropolitana de Alicante-Elche y pese a tener unas características demográficas muy diferentes, también se ha producido un incremento del área urbana. En este caso, la superficie pasa de 2.226 ha a 16.804 ha en el periodo 1956-2005 (Valera Lozano et al., 2013). La primera línea litoral es una zona con un dinamismo muy acentuado, por lo que el crecimiento urbano es representativo en los espacios turísticos de las Islas Baleares, la Costa Blanca, la Costa Brava, Murcia y Almería (González, 2008). La característica diferencial de la costa alicantina y, sobre todo, del litoral de la Región de Murcia es la expansión de los resort, más alejados de la línea de costa y sustituyendo usos agrícolas, principalmente de secano.

Debido a la información que ofrecen en cuanto a datos multitemporales, este tipo de trabajos pueden ser de gran ayuda en la toma de decisiones y ofrecen la posibilidad de ser útiles en la planificación del territorio.

\section{BIBLIOGRAFÍA}

AGUAYO, M., PAUCHARD, A., AZÓCAR, G. y PARRA, O. (2009): «Cambio del uso del suelo en el centro sur de Chile a fines del siglo XX. Entendiendo la dinámica espacial y temporal del paisaje». Revista Chilena de Historia Natural, no 82 (2): 361-374. 
ALONSO SARRÍA, F., GOMARIZ CASTILlO, F. y CÁNOVAS GARCÍA, F. (2010): «Análisis temporal de los cambios de usos del suelo en la cuenca del Segura mediante teledetección. Implicaciones sobre la degradación». Rev. C. \& G., nº 24 (3-4): 73-88.

BELMONTE SERRATO, F., ROMERO DÍAZ, A., RUPÉREZ TIRADO. E. y MORENO BROTÓNS, J. (2011): «El impacto de la agricultura intensiva en el uso turístico de las playas de marina de Cope (Murcia)». Cuadernos de Turismo, $\mathrm{n}^{\circ}$ 27, 23-38.

BELMONTE SERRATO, F., ROMERO DÍAZ, M. A., y RUIZ SINOGA, J.D. (2013): «Retroceso de la línea de costa en playas del sur de la Región de Murcia». Scripta Nova: Revista electrónica de Geografía y Ciencias Sociales, Vol. XVII, núm. 443. Disponible en: http://www.ub.edu/geocrit/sn/sn-443.htm

BOCCO, G., MENDOZA, M., y MASERA, O. (2001): «La dinámica del cambio de uso del suelo en Michoacán». Una propuesta metodológica para el estudio de los procesos de deforestación. Investigaciones Geográficas, nº 44, 18-38.

BURRIEL, E.L. (2008): «La "década prodigiosa" del urbanismo español (19972006)». Scripta Nova. Revista Electrónica de Geografía y Ciencias Sociales, vol. XII, núm. 270 (64). Disponible en: http://www.ub.edu/geocrit/-xcol/383.htm

CALATRAVA LEYVA, J. (2006): Plan estratégico del sector agroalimentario de la Región de Murcia. Análisis del sector de la almendra. Consejería de Agricultura y Agua de la CARM, Universidad de Murcia y Universidad Politécnica de Cartagena.

CANALES MARTÍNEZ, G., y JUÁREZ SÁNCHEZ-RUBIO, C. (1994). «Nuevos regadíos en el secano del Bajo Segura: el modelo referencial de San Onofre-Torremendo (19531992)». Investigaciones Geográficas, $\mathrm{n}^{\circ}$ 12, 215-238.

DUNJÓ G., G. PARDINI y M. GISPERT. (2003): «Land use change effects on abandoned terraced soils in a Mediterranean catchment, NE Spain». Catena, n 52, 23-37.

FAO. (1996) Forest resources assesment 1990. «Survey of tropical forest cover and study of change processes». Roma. FAO forestry paper 130.152 pp. Disponible en: http://www. fao.org/docrep/007/w0015e/w0015e00.htm

KUMMER, D. M. y TURNER, B.L. II (1994): «The human causes of deforestation in Southeast Asia"». Bioscience, n 44, 323-328.

GAJA I DÍAZ, F. (2008): «El "tsunami urbanizador" en el litoral mediterráneo. El ciclo de hiperproducción inmobiliaria 1996-2006». Scripta Nova. Revista Electrónica de Geografía y Ciencias Sociales, vol. XII, núm. 270 (66). Disponible en:

GONZÁLEZ, F. (2008): «El papel de los destinos turísticos en la transformación sociodemográfica del litoral mediterráneo español». Boletín de la Asociación de Geógrafos Españoles, n47, 79-107.

GUTIÉRREZ, J. y GOULD M. 2000. SIG: Sistemas de información geográfica. Editorial Síntesis. Madrid, España.

HERNÁNDEZ HERNÁNDEZ, M. y MORALES GIL, A. (2008): «Trascendencia socioeconómica del trasvase tajo segura tras 30 años de su funcionamiento en la provincia de alicante». Investigaciones Geográficas, n 46, 31-48.

IBARRA MARINAS, D., MARTÍNEZ SALVADOR, A., CONESA GARCIA C. y BELMONTE SERRATO, F. «Estimación de evaporación en balsas de riego mediante el empleo de técnicas de teledetección. Estudio aplicado a la vertiente litoral sur de la Región de Murcia». XVI Congreso Nacional de Tecnologías de la Información Geográfica, Alicante 2014. 
INFRAESTRUCTURA DE DATOS ESPACIALES DE LA REGIÓN DE MURCIA. IDERM. 2015. www.cartomur.imida.es (Fecha de acceso: 25/02/2015)

INSTITUTO GEOGRÁFICO NACIONAL (IGN) 2015. www.ign.es (Fecha de acceso: 25/02/2015)

JANSEN, L.J.M. y DI GREGORIO, A. (2002): «Parametric land cover and land-use classification as tools for environmental change detection». Agriculture, Ecosystems and Environment, $\mathrm{n}^{\circ}$ 91, 89-100.

KOSMAS, C., DANALATOS, N.G., LÓPEZ-BERMÚDEZ, F., y ROMERO DÍAZ, M.A. (2002): «The effect of Land Use and Soil Erosion and Land Degradation under Mediterranean Conditions». En: Mediterranean Desertification. A mosaic of processes and responses (N.A. Geeson, D.J. Brandt y J.B. Thornes eds.). J.Wiley y sons, Chichester, 57-70.

LAMBIN, E.F., TURNER, B.L., GEIST, H.J., AGBOLA, S.B., ANGELSEN, A., BRUCE, J. W., COOMES, O.T., DIRZO, R., FISCHER, G., FOLKE, C., GEORGE, C., HOMEWOOD, K., IMBERNON, J., LEEMANS, R., LI, X., MORÁN, E.F., MORTIMORE, M., RAMAKRISHNAN, P.S., RICHARDS, J.F., SKANES, H., STEFFEN, W., STONE, G.D., SVEDIN, U., VELDKAMP, T.A., VOGEL, C., y XU, J. (2001): «The causes of land-use and land-cover change: moving beyond the myths». Global Environmental Change, $\mathrm{n}^{\mathrm{o}}$ 11, 261-269.

LÓPEZ-BERMÚDEZ, F., SÁNCHEZ-FUSTER, M.C. y ROMERO-DÍAZ, A. (1995): «Incidencia de los modelos de gestión socio-económica (siglos XIX y XX) en la degradación del suelo en el Campo de Lorca (Cuenca del Guadalentín, Murcia)». Papeles de Geografía, nº 22, 5-18.

LÓPEZ, E., BOCCO, G., MENDOZA, M., y DUHAU, E. (2001): «Predicting land-cover and land-use change in the urban fringe - A case in Morelia city, México». Landscape and Urban Planning, nº55, 271-285.

MATSON, P.A., PARTON, W.J., POWER, A.G., y SWIFT, M.J. (1997): «Agricultural intensification and ecosystem properties». Science, $\mathrm{n}^{\circ}$ 277, 504-509.

MIR-GUAL, M. (2009): «Modificaciones del perfil de playas en las Islas Balear (Playas de Can Picafort y Es Comú de Muro)». Investigaciones Geográficas, nº 50, 191-207

MORALES GIL, A. (Coord.) (2003): Cultura, paisajes y sociedades en el eje de desarrollo territorial del Bajo Segura y Campo de Cartagena, Murcia, AUSUR.

PASCUAL AGUILAR, J.A. (2004): «Dinámica reciente de usos del suelo en el continuo metropolitano de valencia». Cuadernos de Geografía, nº 76, 183-202.

PEÑA, J., PÓVEDA, R.M., BONET, A., BELLOT, J. y ESCARRÉ, A. (2005): «Cartografía de las coberturas y usos del suelo de la Marina Baixa (Alicante) para 1956, 1978 y 2000». Investigaciones Geográficas, $\mathrm{n}^{\circ} 37$ 93-107.

RODRIGO, J., SENCIALES GONZÁLEZ, J. M. y FERRÉ BUENO, E. (2014): «Análisis de los cambios de usos del suelo en el área de Casapalma (Valle del Guadalhorce, Málaga) entre 1991 y 2007». Papeles de Geografía, n $59,157-171$.

RODRÍGUEZ LÓPEZ, J. (2006): «Los booms inmobiliarios en España. Un análisis de tres períodos». Papeles de Economía Española, nº 109, 76-90.

ROMERO DÍAZ, A., IBARRA MARINAS, D., BELMONTE SERRATO, F. y RUIZ SINOGA, J.D. (2012): «Erosión en las playas de "Las Covaticas" y "Parazuelos" en el litoral de la Región de Murcia». In A. González Díaz (Ed.) Avances de la geomorfología en España 2012-2012. Santander: Universidad de Cantabria, pp. 379-383. 
ROSETE VERGÉS, F.A., PÉREZ DAMIÁN, J.L. y BOCCO, G. (2009): «Contribución al análisis del cambio de uso del suelo y vegetación (1978-2000) en la Península de Baja California, México». Investigación Ambiental, nº 1(1), 70-82.

SEISDEDOS, J., MULAS, J., GONZALEZ DE VALLEJO, L. I., RODRIGUEZ FRANCO, J. A., GRACIA, F. J., DEL RIO, L., y GARROTE, J. (2013): «Estudio y cartografia de los peligros naturales costeros de la Región de Murcia». Boletin Geologico y Minero, $\mathrm{n}^{\circ}$ $124(3), 505520$.

SKOLE, D. L., H. CHOMENTOWSKI, W. A. SALAS y NOBRE A. D. (1994): «Physical and human dimensions of deforestation in Amazonia». Bioscience, $\mathrm{n}^{\circ}$ 44, 314-322.

TURNER, M.G., ARTHAUD G.J. y ENGSTROM, R.T. (1995): «Usefulness of espatially explicit population models in land management». Ecological Applications, no 5 (1), 12-16.

VALERA LOZANO, A., AÑÓ VIDAL, C. y SÁNCHEZ DÍAZ, J. J. (2007) «Crecimiento urbano (1956-1998) en el entorno metropolitano de Alacant-Elx (Comunidad Valenciana)». Boletín de la Asociación de Geógrafos Españoles, nº 44, 169-186.

VERA, F. (2005): «El auge de la función residencial en destinos turísticos del litoral mediterráneo: entre el crecimiento y la renovación». Papers de Turisme, nº 37, 95-116. 
